Pacific Journal of Mathematics

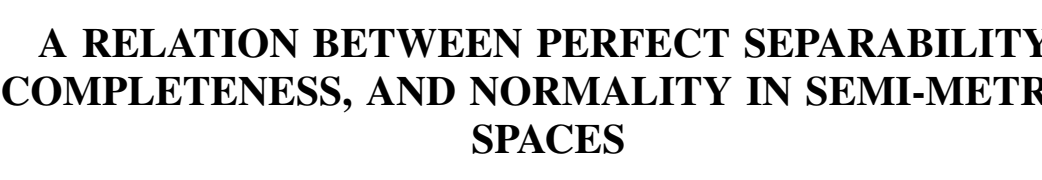




\title{
A RELATION BETWEEN PERFECT SEPARABILITY, COMPLETENESS, AND NORMALITY IN SEMI-METRIC SPACES
}

\author{
LOUIS F. MCAULEY
}

1. Introduction. This paper proves that a regular semi-metric ${ }^{1}$ topological space $S$ may have such properties as hereditary separability, collectionwise normality [1], paracompactness [10], and weak completeness without being either a developable space [1] or a metric space. However, if $S$ is strongly complete, then hereditary separability implies perfect separability [12] and consequently metrizability. It has been proved $[1 ; 12]$ that a regular developable topological space (Moore space) is metrizable provided that it is perfectly separable. Thus, a regular semi-metric topological space may be far removed from a Moore space contrary to a result announced by C. W. Vickery [11]. The notion of $p$-separability due to Frechet is generalized and a question raised by $\mathrm{W}$. A. Wilson [14, p. 336] is answered in the affirmative. Throughout this paper, $S$ denotes a regular semi-metric topological space.

\section{Weak and strong completeness.}

Definition 2.1. A space $S$ is said to be $\left\{\begin{array}{c}\text { weakly complete } \\ \text { strongly complete }\end{array}\right\}$ provided there exists a distance function $d$ such that (1) the topology of $S$ is invariant with respect to $d$ and (2) if $\left\{M_{i}\right\}$ is a monotonic decending sequence of closed subsets of $S$ such that, for each $i$, there exists a $1 / i$-neighborhood of a point $p_{i}\left\{\begin{array}{c}\text { in } M_{i} \\ \text { in } S\end{array}\right\}$ which contains $M_{i}$, then $\Pi M_{i}$ contains a point.

It is now shown that strong completness is sufficient to bridge a gap between a hereditarily separable space $S$ and a developable space.

Received August 13, 1954 and in revised form April 15, 1955. Presented to the American Mathematical Society, April 24, 1954. The author wishes to express appreciation to Professor F. B. Jones for having stimulated this research in classes at The University of North Carolina.

1 A topological space $S$ is said to be a semi-metric topological space provided there is a distance function $d$ defined for $S$ such that (1) if each of the letters $x$ and $y$ denotes a point of $S$, then $d(x, y)=d(y, x)$ denotes a non-negative number, (2) $d(x, y)=0$ if and only if $x=y$, and (3) the topology of $S$ is invariant with respect to the distance function $d$, that is, if $p$ is a limit point of a subset $M$ of $S$, then $p$ is a distance limit point of $M$ and conversely. As usual, $S$ is said to be regular provided that if $R$ is an open set containing a point $p$ of $S$, then there exists an open set $D$ such that $R \supset \bar{D} \supset p$. A topological space $\left(T_{1}\right)$ is defined as in [9]. 
THEOREM 2.2. Every hereditarily separable and strongly complete space $S$ is perfectly separable.

Proof. Let $d$ denote a semi-metric for the space $S$. For each pair of natural numbers $h$ and $k$, let $M_{k k}$ denote the set of all points $p$ such that for some open set $R$, the spherical neighborhoods $U_{1 / h}(p)$ and $U_{1 / k}(p)$ satisfy $U_{1 / n}(p) \supset \bar{R} \supset R \supset U_{1 / k}(p)$. It should be noted that the spherical neighborhoods defined by $d$ may fail to be open sets. Since $S$ is hereditarily separable, there exists a countable dense subset $N_{h k}$ of $M_{h k}$. Let $G_{h k}$ denote a countable collection of open sets such that for each point $p$ in $N_{h k}$, there exists an open set $R$ in $G_{h k}$ such that $U_{1 / n}(p) \supset \bar{R}$ $\supset R \supset U_{1 / k}(p)$. Clearly, $G_{h k}$ covers $M_{h k}$. Furthermore, each point of $S$ lies in $M_{h k}$ for some $h$ and $k$.

Let $G$ denote a countable collection of open sets covering $S$ such that (1) the intersection of two elements of $G$ is an element of $G$ and (2) if $Q$ is an element of $G_{h k}$ for some $h$ and $k$, then $Q \in G$. The collection $G$ is a basis for $S$. For, suppose that there exists an open set $R$ containing a point $p$ such that there exists no element of $G$ that contains $p$ and lies in $R$. Then, for each $i$, there exists an integer $k_{i}$ and an element $R_{i}$ of $G_{i k_{i}}$ which contains $p$ such that $\prod_{j=1}^{i} R_{j}$ fails to lie in $R$. Now, there exists a point $p_{i}$ such that $U_{1 / i}\left(p_{i}\right) \supset \bar{R}_{i} \supset \prod_{j=1}^{i} \bar{R}_{j} \cdot(S-R)$ $=M_{i}$. Since $M$ is strongly complete, $I M_{i}$ contains a point $q \neq p$. Thus, $d\left(p_{i}, q\right)<1 / i$ and $d\left(p_{i}, p\right)<1 / i$ for each $i$. This is impossible. Hence, $S$ is perfectly separable.

It is an interesting fact that Cauchy completeness, when defined in a natural way for a space $S$ (see [9] and footnote 2), is equivalent to weak completeness in $S$.

THEOREM 2.3. A necessary and sufficient condition that a semimetric space $S$ be weakly complete is that every Cauchy sequence ${ }^{3}$ of points of $S$ have a limit point in $S$.

Proof. The condition is necessary. Suppose that there exists a Cauchy sequence $\left\{p_{i}\right\}$ of points of $S$ which has no limit point in $S$. Thus, there exists a subsequence $\left\{p_{n_{i}}\right\}$ of $\left\{p_{i}\right\}$ such that for each $i$,

2 This theorem was proved independently by my classmate Wyman Richardson in one of F. B. Jones' classes.

3 A Cauchy sequence $\left\{p_{i}\right\}$ of points is said to have a limit point $p$ provided that there exists a subsequence $\left\{p_{n_{i}}\right\}$ of $\left\{p_{i}\right\}$ which converges to $p$. There exists a Cauchy sequence of points in a space $S$ which has a limit point but which has no sequential limit point. 
$U_{1 / i}\left(p_{n_{i}}\right) \supset p_{n_{j}}$ for $j \geqq i$. Let $M_{i}=\sum_{j=i}^{\infty} p_{n_{j}}$. Since $\Pi M_{i}=0$, there is a contradiction to the hypothesis that $S$ is weakly complete.

The condition is sufficient. Suppose that $\left\{M_{i}\right\}$ denotes a monotonic descending sequence of closed subsets of $S$ such that for each $i$, there exists a point $p_{i}$ such that $p_{i} \in M_{i} \subset U_{1 / i}\left(p_{i}\right)$. Since $\left\{p_{i}\right\}$ is a Cauchy sequence, the set $\Pi M_{i}$ contains a point $p$.

3. Non-equivalence of regular semi-metric topological spaces and regular developable (Moore) spaces. Many theorems which are true for Moore spaces have analogues which hold for regular semi-metric topological spacest. However, the fact that a regular semi-metric topological space $S$ is far removed from a Moore space is stressed by the following examples and theorems. From these, it follows that the condition of either separability or screenability for the metrization of a normal Moore space due to Jones [5] and Bing [1], respectively, has no analogue which holds in a normal space $S$.

Consider the following example of a regular semi-metric space which is not a Moore space. Some additional properties of this space are given in Theorem 3.2 .

ExAmple 3.1. Let $X$ denote the $x$-axis of the Cartesian plane $E^{2}$. A semi-metric $D(p, q)$ will be defined for $E^{2}$ in the following way. Suppose that each of the letters $p$ and $q$ denotes a point of $E^{2}$. If $X$ contains both or neither of the points $p$ and $q$, then define $D(p, q)$ to be the Cartesian distance $d(p, q)$. If $p \in X$ and $q \notin X$, then define $D(p, q)$ to be $d(p, q)+\alpha$ where $\alpha$ is a non-obtuse angle (measured in radians) between $X$ and the line $L$ determined by $p$ and $q$. If $p \notin X$ and $q \in X$, define $D(p, q)$ to be $D(q, p)$. Clearly, $D$ is a semi-metric for $E^{2}$. For each positive integer $n$ and each point $p, U_{1 / n}(p)$ is defined to be an open set provided that either $p \in X$ or $U_{1 / n}(p)$ lies in one of the two components of $E^{2}-X$. Considering the open sets defined in this way as the elements of basis for a topology, $E^{2}$ becomes a regular connected and locally connected semi-metric topological space $S$ which is not a Moore space. It should be noted that $S$ is hereditarily separable since it is the sum of two hereditarily separable sets $S-X$ and $X$.

THEOREM 3.2. There exists a connected and locally connected regular semi-metric topological space $S$ which is hereditarily separable, weakly complete, strongly screenable [1], collectionwise normal, completely normal, and paracompact but which is neither perfectly separable nor a Moore space nor metrizable.

${ }^{4}$ This is included in unpublished work of F. B. Jones. 
Proof. Let $S$ be the space $E^{2}$ with the topology defined in Example 3.1. The space $S$ is not metrizable since it is not a Moore space.

Suppose that $S$ is perfectly separable. Then there exists a countable collection $H$ of spherical neighborhoods in $S$ that defines the topology of $S$. For each number $e>0$ and each point $p$ in $X, U_{e}(p)$ contains an element $h(e, p)$ of $H$. By the definition of $U_{e}(p)$, it follows that the center of the spherical neighborhood $h(e, p)$ is $p$. This is impossible since $H$ is countable and $X$ is uncountable.

In order to show that $S$ is weakly complete, a distance function $E$ different from that given in Example 3.1 will be introduced. Let $L_{1}$ and $L_{2}$ be two distinct lines parallel to and at a unit distance from $X$, and denote by $C(X)$ the component of $S-\left(L_{1}+L_{2}\right)$ that contains $X$. For any pair of points $p$ and $q$ of $C(X)-X$, define $E(p, q)$ to be $d(p, q) /$ $d(X, p) d(X, q)$, where $d$ is the ordinary Cartesian distance function. If either of two points $p$ and $q$ fails to lie in $C(X)-X$, then define $E(p, q)$ to be $D(p, q)$ as given in Example 3.1. It follows that the topology of $S$ is unchanged by $E$. In the remainder of this paragraph, the spherical neighborhoods considered will be those defined by $E$. Now, suppose that $\left\{M_{i}\right\}$ is a monotonic descending sequence of closed point sets and $\left\{p_{i}\right\}$ is a sequence of points such that for each $i, p_{i} \in M_{i} \subset U_{1 / i}\left(p_{i}\right)$. If there exists a subscript $n$ such that $X \cdot M_{n}=0$, then $X \cdot M_{i}=0$ for $i>n$. From this it follows that there exists $m>n$ such that $U_{1 / m}\left(p_{m}\right) \cdot X=0$. For, suppose that this is not the case. Then there must exist a subsequence $\left\{p_{n_{i}}\right\}$ of $\left\{p_{i}\right\}$ such that $\left\{d\left(X, p_{n_{i}}\right)\right\}$ converges to 0 . Consequently, by the definition of $E$, the sequence $\left\{E\left(p_{n}, p_{n_{i}}\right)\right\}$ of real numbers is unbounded. This is contrary to the assumption that $U_{1 / n}\left(p_{n}\right) \supset M_{n}$. Thus, the existence of the required integer $m$ is established. It follows that $\Pi M_{i} \neq 0$ in this case. For the remaining case, suppose that for each $i, X \cdot M_{i} \neq 0$. Since $\left\{X \cdot M_{i}\right\}$ is a bounded monotonic descending sequence of non-empty closed subsets of $X$, it follows that $\Pi M_{i} \neq 0$. Hence, $S$ is weakly complete.

The space $S$ is strongly screenable. Consider the metric subspaces $S-X$ and $X$ of $S$. These are strongly screenable by theorems due to Bing [1]. Let $G$ denote an open covering of $S$. Denote by $H$ and $K$ open coverings of $X$ and $S-X$, respectively, such that for $g$ in $G, g \cdot X \in H$ and $g \cdot(S-X) \in K$. There exists a sequence $\left\{H_{i}\right\}$ of discrete collections [1] of open intervals of $X$ such that $\Sigma H_{i}$ covers $X$ and for each $i, H_{i}$ is a refinement of $H$. Let $I$ deote an interval in $H_{i}$ for some $i$. Since $\bar{I}$ contains no point of the closure of $\left(H_{i}-I\right)^{*}$ [the logical sum of the elements of $\left.H_{i}-I\right]$ and $I$ lies in some element $g$ of $G$, it follows that there exist discrete collections $P$ and $Q$ of $1 / n$-neighborhoods of points in $X$ such that (1) each element of $P$ and each element of $Q$ lies in $g$, (2) the closure of no element of either $P$ or $Q$ intersects the closure of 
$\left(H_{i}-I\right)^{*}$, and (3) $P+Q$ covers $I$. It follows that there exists a sequence $\left\{X_{i}\right\}$ of discrete collections each of which is a refinement of $G$ and such that $\Sigma X_{i} \supset X$. Similarly, there exists a sequence $\left\{K_{i}\right\}$ of discrete collections each of which is a refinement of $K$ and such that $\Sigma K_{i} \supset S-X$. For each natural number $i$, let $G_{2 i}=X_{i}$ and $G_{2 i-1}=K_{i}$. Thus, $\left\{G_{i}\right\}$ is a sequence of discrete collections of open subsets of $S$ such that $\Sigma G_{i}$ covers $S$ and $G_{i}$ refines $G$ for each $i$. Hence, $S$ is strongly screenable.

Now $S$, being a regular strongly screenable topological space, is collectionwise normal [1]. It also follows that $S$ is paracompact by a theorem due to Ernest Michael [6].

To complete the proof of theorem 3.2, it must be shown that $S$ is completely normal. It has been proved by F. B. Jones [5] that every normal Moore space is completely normal ${ }^{5}$. A simple modification of his argument shows that every normal semi-metric topological space is completely normal. This completes the proof.

Mary E. Estill [3] has considered complete Moore spaces in any one of three definitions of completeness. Perhaps intuition would lead one to suspect that a complete Moore space, in one of these senses, would be strongly complete. The following example and theorem shows that this is not the case. As a matter of fact, in a Moore space, the concept of strong completeness is more restrictive than that of completeness.

ExAmple 3.3. Let $X$ denote the $x$-axis of the Cartesian plane $E^{2}$. A semi-metric $D(p, q)$ will be defined for $E^{2}$ in the following way. Suppose that $p$ and $q$ are two distinct points of $E^{2}$. If neither $p$ nor $q$ lies in $X$, then define $D(p, q)$ to be $d(p, q)$ where $d$ is the ordinary Cartesian metric. If $p \in X$, then let $D(p, q)=d(p, q)+\alpha$ where $\alpha$ is an angle (measured in radians) between a line $L_{1}$ containing $p+q$ and a vertical line $L_{2}$ containing $p$ such that $0 \leqq \alpha \leqq \pi / 2$. If $D(q, p)$ is not defined above, then let $D(q, p)=D(p, q)$. For $p$ in $X$, let $D(p, p)=0$. Clearly, $D$ is a semi-metric for $E^{2}$. For each point $p$ in $E^{2}$ and each natural number $n, U_{1 / n}(p)$ is defined to be an open set. With this definition of open sets, $E^{2}$ becomes a regular connected and locally connected semi-metric topological space $S$. It should be noted that $S$ is separable but not hereditarily separable.

THEOREM 3.4. There exists a complete Moore space $S$ which is not strongly complete.

Proof. Let $S$ be the space $E^{2}$ with the topology defined in Example

5 A space $S$ is said to be completely normal provided that for two mutually separate subsets $H$ and $K$ of $S$ there exists matually exclusive open coverings of $H$ and $K$. See [5]. 
3.3. It will first be shown that $S$ is not strongly complete.

Suppose that $S$ is strongly complete. Then there exists a semimetric $E$ defined for $S$ such that (1) the topology of $S$ is unchanged by $E$ and (2) if $\left\{M_{i}\right\}$ is a monotonic descending sequence of closed subsets of $S$ such that for each $i$ and some point $p_{i}$ in $S, U_{1 / i}\left(p_{i}\right) \supset M_{i}$, then $\Pi M_{i} \neq 0$. It should be noted that the spherical neighborhoods defined by $E$ may fail to be open sets.

Consider an interval $A$ of $X$. For each pair of natural numbers $h$ and $k$, let $M_{h k}$ denote the subset of $A$ of all points $p$ such that for some open set $R, U_{1 / n}(p) \supset R \supset U_{1 / k}(p)$. For some natural number $h_{1}$, the set $M_{1 h_{1}}$ is uncountable. Now, $M_{1 h_{1}}$ contains an uncountable subset $N_{1 h_{1}}$ such that

(1) there exists a line $L_{1}$ parallel to $X$ where $d\left(L_{1}, X\right) \leqq 1$ and

(2) for each point $p$ in $N_{1 h_{1}}$, there exists an open set $R(p)$ where $U_{1}(p) \supset R(p) \supset U_{1 / h_{1}}(p)$ such that $R(p)$ contains an interval $I$ of $L_{1}$ whose length (in the Cartesian sense) is greater than a positive number $e_{1}$ and which has as its center a point $q$ whose projection on $X$ is $p$.

Now there exists an integer $h_{2}>h_{1}$ such that $N_{1 h_{1}}$ contains an uncountable subset $N_{2 h_{2}}$ such that

(1) there exists a line $L_{2}$ parallel to $X$ where $d\left(L_{2}, X\right) \leqq 1 / 2$ and

(2) for each point $p$ in $N_{2 h_{2}}$, there exists an open set $R(p)$ where $U_{1 / 2}(p) \supset R(p) \supset U_{1 / h_{2}}(p)$ such that $R(p)$ contains an interval $I$ of $L_{2}$ whose length is greater than a positive number $e_{2}$ and which has as its center a point $q$ whose projection on $X$ is $p$.

If follows that there exists a monotonic descending sequence $\left\{N_{i n_{i}}\right\}$ of subsets of $A$ and a sequence $\left\{L_{i}\right\}$ of lines parallel to $X$ and converging to it such that for each $i$, if $p_{i}$ is a point of $N_{i h_{i}}$, there exists an open set $R\left(p_{i}\right)$ where $U_{1 / i}\left(p_{i}\right) \supset R\left(p_{i}\right) \supset U_{1 / h_{i}}\left(p_{i}\right)$ such that $R\left(p_{i}\right)$ contains an interval $I_{i}$ of $L_{i}$ whose length is greater than a positive number $e_{i}$ and which has as its center a point $q_{i}$ whose projection on $X$ is $p_{i}$. Since $A$ is a compact subset of $E^{2}$ there exists a monotone sequence $\left\{p_{i}\right\}$ of points converging to a point $p$ in $A$ such that for each $i, p_{i} \in N_{i h_{i}}$. Let $L$ be a vertical line containing $p$, and for each $i$, define $x_{i}=L \cdot L_{i}$. It follows that there exists a monotonic increasing sequence $\left\{k_{i}\right\}$ of natural numbers such that for each $i, E\left(x_{i}, p_{j}\right)<1 / i$ for all $j>k_{i}$. The set $M_{i}=\sum_{k=k_{i}}^{\infty} p_{k}$ is closed in $S$ for each $i$ and $U_{1 / i}\left(x_{i}\right) \supset M_{i}$. It follows that $\Pi M_{i}=0$.

This is contrary to the assumption that $S$ is strongly complete.

It now remains to be shown that $S$ is a complete Moore space. For a point $p$ in $X$, there exists a sequence $\left\{R_{i}\right\}$ of open sets closing down ${ }^{6}$

${ }^{6}$ A sequence of open sets $\left\{R_{i}\right\}$ is said to close down on a point $p$ if for each $i$, $R_{i} \supset \bar{R}_{\imath+1}$ and $\Pi R_{i}=p$. 
on $p$. On the other hand, if $p$ denotes a point of $S-X$, there exists a sequence $\left\{R_{i}\right\}$ of open sets closing down on $p$ such that for each $i, R_{i} \cdot X=0$. With each point $p$ of $S$, associate exactly one such sequence $\left\{R_{i}\right\}$. For each $i$, let $G_{i}$ denote the collection of all open sets $R$ such that for some point $p$ of $S, R$ is the $j$ th member of the sequence associated with $p$, and $j \geq i$. It follows that $S$ is a complete Moore space.

4. A question due to W. A. Wilson. An affirmative answer is given in this section to a question raised by Wilson [14, p. 366] in 1931 . The following axioms and definitions [14] are listed for convenience.

A set $Z$ is said to be a (Menger) semi-metric space provided that corresponding to each pair of points $(a, b)$ of $Z$, there is a non-negative real number $d(a, b)$ satisfying the following axioms:

Axiom I. $d(a, b)=d(b, a)$.

Axiom II. $d(a, b)=0$ if and only if $a=b$.

Wilson has introduced the following additional axiom:

Axiom $W$. For each point $a$ and each positive number $k$, there is a positive number $r$ such that if $b$ is a point for which $d(a, b) \geqq k$ and $c$ is any point, then $d(a, c)+d(b, c) \geq r$.

Now, let $r=f(a, k)$ denote the largest $r$ such that $d(a, c)+d(b, c) \geqq r$ in Axiom $W$. For each point $a$ and each positive number $k$, let $r=f(a, k)$, $r_{1}=f(a, r)$, and $r_{2}$ denote a positive number such that $r_{2}<r_{1}$. Wilson calls the set $\sigma$ of points $x$ such that $d(a, x)<r_{2}$ an inner sphere, with center $a$, corresponding to $a$ and $k$.

THEOREM 4.1. Suppose that $Z$ denotes a separable semi-metric space satisfying Axiom $W$. If $d$ denotes a distance function defined for $Z$ which leaves limit points invariant, then there exists a countable dense subset $E=\Sigma p_{i}$ of $Z$ such that for any positive number $k$, each point $p$ of $Z$ lies in an inner sphere $\sigma$ corresponding to $p_{i}$ and $k$ for some natural number $i$.

Proof. By a corollary due to Wilson [14], $Z$ is homeomorphic to a metric space. Since a separable metric space is hereditarily separable, it follows that $Z$ is hereditarily separable.

Let $S_{e}(p)$ denote a spherial neighborhood in $Z$. For each pair of natural numbers $h$ and $k$, let $M_{h k}$ denote the set of all points $p$ such that there exists an inner sphere $\sigma$ corresponding to $1 / h$ and $p$ such that $S_{1 / h}(p) \supset \sigma \supset S_{1 / k}(p)$. Since $Z$ is hereditarily separable, $M_{h k}$ contains a countable dense subset $N_{h k}$. Let $K_{h k}$ be a countable collection of inner spheres such that if $p \in N_{h k}$, then there exists an inner sphere $\sigma$ in $K_{h k}$ corresponding to $1 / h$ and $p$ such that $S_{1 / h}(p) \supset \sigma \supset S_{1 / k}(p)$. It 
follows that $K_{h i}$ covers $M_{h k_{i}}$. Denote by $E=\Sigma_{i} p_{i}$, the countable dense subset $\sum_{h, k=1}^{\infty} N_{h k}$ of $Z_{h}$.

The set $E$ satisfies the conclusion of Theorem 4.1. For, if $c$ is any positive number, there exists a positive integer $h$ such that $1 / h<c$. Also, for $p$ in $Z$, there exists $k$ such that $p \in M_{h k}$. Since $K_{h k}$ covers $N_{l l i}$, there exists an inner sphere $\sigma$ corresponding to $p_{i}$ and $1 / h$ for some $i$ such that $\sigma \supset p$. Hence, the inner sphere $\sigma_{1}$ which corresponds to $p_{i}$ and $c$ contains $\sigma$ and $p$.

Now Wilson's question referred to above is answered.

5. Generalized Frechet $\boldsymbol{p}$-separability. The following definition is a natural generalization of the notion of $p$-separability [4]. It is proved that in a space $S$ this notion is equivalent to hereditary separability.

Definition 5.1. A regular semi-metric topological space $S$ (or semimetric space $Z$ ) is said to be $p$-separable provided that

(1) given any distance function $d$ which leaves limit points invariant and

(2) given any collection $H$ of subsets of $S$ which has the property that for each number $k>0$ and each point $p$ of $S$, there exists $h$ in $I$ such that $U_{k}(p) \supset h \supset U_{e}(p)$ for some positive number $e$,

then there exists a countable dense subset $E=\Sigma p_{i}$ such that for each positive number $f$, each point $p$ of $S$ lies in an element $h$ of $H$ such that $U_{f}\left(p_{i}\right) \supset h \supset p_{i}$ for some $i$.

The following theorem may be proved in a manner analogous to that used in the proof of Theorem 4.1.

THEOREM 5.2. Every hereditarily separable semi-metric space $Z$ is $p$-separable.

THEOREM 5.3. A necessary and sufficient condition that a regular semi-metric topological space $S$ be hereditarily separable is that $S$ be $p$ separable.

Proof. The necessity of the condition follows from Theorem 5.2.

It will now be shown that the condition is sufficient. Suppose that $d$ denotes a semi-metric for $S$, and that $S$ is not hereditarily separable. Then $S$ contains an uncountable subset $N$ which has no limit point in $S$. Now, consider a semi-metric $D$ defined in the following way. For each $i$, let $D_{i}$ denote the set of all points $x$ of $S$ such that for some point $p$ in $N, x$ lies in an open set $R \subset u_{1 / i}(p)$ where $u_{1 / i}(p)$ is a spherical neighborhood defined by $d$. Thus, $\left\{D_{i}\right\}$ is a monotonic descending sequ- 
ence of open sets such that $I I D_{\imath}=N$. For each $i$ and each point $p$ in $D_{i}-N$, associate exactly one open set $R_{c}(p)$ containing $p$ and lying in $D_{i}$ such that for some number $e, u_{e}(p) \supset \bar{R}(p)$ and $u_{e}(p) \cdot N=0$. If $x$ and $y$ denote points of $S-N$ such that for some $i, D_{i} \supset x+y$ and $D_{i+1} \not \supset x+y$, then define $D(x, y)$ to be $i$ provided that $R_{i}(x) \not \supset y$ and $R_{i}(y) \not \supset x$. For points $x$ and $y$ of $S$ for which $D(x, y)$ is not defined above, let $D(x, y)=d(x, y)$. It follows that limit points are invariant with respect to $D$.

Next, let $H$ denote a collection of open sets such that for each natural number $i$ and each point $p$ in $S$, there exists $h$ in $H$ such that $U_{1 / i}(p) \supset h \supset p$ where $U_{1 / i}(p)$ is a spherical neighborhood defined by $D$. Since $S$ is $p$-separable, there exists a countable dense subset $E=\Sigma p_{i}$ of $S$ such that for each positive number $f$, each point $p$ of $S$ lies in an element $h(p)$ of $H$ such that for some $i, U_{f}\left(p_{i}\right) \supset h(p)$. There exists an uncountable subset $M$ of $N-E$ and a natural number $t$ such that if $x$ is a point and $D(x, M)<1 / t$, then $x$ lies in $D_{t}$. Let $p \in M$. Then there exist

(1) a number $e>0$ such that $D(p, N-p)=d(p, N-p)>e$,

(2) a positive integer $n$ such that $1 / n<$ smaller $[e, 1 / t]$,

(3) $h$ in $H$,

(4) an integer $i$ such that $U_{1 / n}\left(p_{i}\right) \supset h \supset p$ [thus, $p_{i} \in D_{t}$ ],

(5) an integer $m \geqq t$ such that $p_{i} \in D_{m}-D_{m+1}$,

(6) an open set $R_{m}\left(p_{i}\right)$ associated with $p_{i}$ and $D_{m}$ such that for some number $c, u_{c}\left(p_{i}\right) \supset \bar{R}_{m}\left(p_{i}\right)$ and $u_{c}\left(p_{i}\right) \cdot N=0$,

(7) a positive number $z$ such that for $q$ in $S-R_{m}\left(p_{i}\right), d\left(p_{i}, q\right)>z$,

(8) $x \in h \cdot D_{m}-\left[R_{m}(p)+N\right]$ such that $D(p, x)<z$, and

(9) an open set $R_{m}(x)$ associated with $x$ and $D_{m}$ such that for some number $b, u_{b}(x) \supset \bar{R}_{m}(x)$ and $u_{b}(x) \cdot N=0$.

Therefore, $b<z$. Consequently, $R_{m}(x) \not \supset p_{i} . \quad$ By definition, $D\left(x, p_{i}\right)$ $=m>1 / n$. This is impossible since $U_{1 / n}\left(p_{i}\right) \supset h \supset p+x$. Hence, $S$ is hereditarily separable.

It follows from Theorem 3.2 that $S$ may fail to be either perfectly separable or a metric space.

6. Conditions for semi-metric, regular developable (Moore), and metric spaces. Consider the following three conditions on a topological space $T$.

A. There exists a sequence $\left\{H_{i}\right\}$ such that (a) for each $i, H_{i}$ is a collection of open subsets of $T$, (b) if $p$ is a point and $R$ is an open set containing $p$, then there exists an integer $n$ such that $H_{n}$ contains exactly one element $g(p)$ associated with $p$ such that $R \supset g(p) \supset p$ and 
(c) if $n$ is an integer and $\left\{g_{i}\left(p_{i}\right)\right\}$ is a sequence such that for each $i, g_{i}\left(p_{i}\right)$ belongs to $H_{n}$ and is associated with $p_{i}$, then $\Sigma p_{i}$ has no limit point in $T-\Sigma g_{i}\left(p_{i}\right)$.

B. If $p$ is a point and $R$ is an open set containing $p$, then there exists an integer $n$ such that for $m>n$, each element $g$ of $H_{m}$ which contains $p$ has the property that $R \supset \bar{g}$.

C. For each $i$, the sum of the closures of any subcollection of $H_{i}$ is closed.

THEOREM 6.1. A necessary and sufficient condition that a topological space $T$ be semi-metric is that $T$ satisfy Condition $A$.

Proof. It will first be shown that the condition is sufficient. It follows from Condition A that $T$ satisfies the first axiom of countability. Consider a semi-metric $d$ defined as follows. For two distinct points $p$ and $q$ of $T$, denote by $i$ the least integer such that $H_{i}$ contains an element $g(p)$ associated with $p$ but not containing $q$. Similarly, let $j$ denote the least integer such that $H_{j}$ contains an element $g(q)$ associated with $q$ but not containing $p$. Define $d(p, q)$ to be $1 / \min (i, j)$. For each point $p$, define $d(p, p)$ to be 0 .

Limit points are invariant with respect to $d$. For suppose that $p$ is a limit point (defined by the open sets of $T$ ) of a subset $M$ of $T$ and that $p$ is not a distance limit point of $M$. Then there exists a sequence $\left\{p_{i}\right\}$ of points of $M-p$ which converges to $p$ such that for some integer $n$ and each $i, d\left(p, p_{i}\right)>1 / n$. Thus, there exists an integer $m$, such that, for infinitely many integers $i$, either (1) $H_{m}$ contains $g_{m}(p)$ and $g_{m}(p) \not \supset p_{i}$ or (2) $H_{m}$ contains $g_{m}\left(p_{i}\right)$ and $g_{m}\left(p_{i}\right) \not \supset p$. Since $\left\{p_{i}\right\} \rightarrow p$, (1) is impossible. By Condition A, (2) is impossible. Hence, $p$ is a distance limit point of $M$. It also follows easily that a distance limit point of a subset $M$ of $T$ is an open set limit point of $M$. This completes the proof of the sufficiency.

The condition is necessary. For each point $p$ and each pair of natural numbers $h$ and $k$, let $R_{h k}(p)$ denote an open set when it exists, such that $U_{1 / h}(p) \supset R_{h k}(p) \supset U_{1 / k}(p)$. With $h, k$, and $p$ associate exactly one such open set, and let $G_{k k}$ denote the corresponding collection of open sets for each point $p$ in $T$. There exists a sequence $\left\{H_{i}\right\}$ such that there is a one to one correspondence between the elements of $\left\{H_{i}\right\}$ and the elements of $\left\{G_{n m}\right\}$. It follows that $\left\{H_{i}\right\}$ satisfies Condition A.

As Example 3.1 illustrates, a regular semi-metric topological space may fail to be a Moore space.

THEOREM 6.2. A necessary and sufficient condition that a topological space $T$ be a Moore space is that $T$ satisfy Conditions $A$ and $B$. 
Proof. The condition is sufficient. For each positive integer $i$, let $G_{i}=\sum_{j=i}^{\infty} H_{j}$. If the word "region" is interpreted as "open set," then it follows that Axioms 0 and 1 (1)-(3) due to Moore [7] are satisfied.

The condition is necessary. It will be shown first that $T$ is a semimetric topological space. Let $p$ and $q$ be distinct points of $T$. Denote by $n$ the least positive integer such that if $g(p)$ and $g(q)$ are regions in $G_{n}$ containing $p$ and $q$, respectively, then $g(p) \cdot g(q)=0$. Note that $\left\{G_{i}\right\}$ is given by Axiom 1 of [7]. Consequently, define $d(p, q)$ to $1 / n$. It follows that $d$ is a semi-metric distance function and that limit points are invariant with respect to $d$. By Theorem 6.1, T satisfies Condition A.

Now, define $\left\{H_{i}\right\}$ in a manner described in the proof of Theorem 6.1 with the additional requirement that $R_{h k}(p)$ lie in a region of $G_{h}$. It follows that $\left\{H_{i}\right\}$ satisfies Conditions $\mathrm{A}$ and $\mathrm{B}$.

THEOREM 6.3. A necessary and sufficient condition that a topological space $T$ be metric is that it satisfy Conditions $\mathrm{A}, \mathrm{B}$, and $\mathrm{C}$.

A proof of Theorem 6.3 follows by use of Bing's Theorem 4 of [1] and Theorem 6.1 above.

Question. Is it possible to partition either Bing's Theorem 4 of [1] or Moore's metrization theorem [8; 13], stated below, into three or more parts which begins with a condition for a topological space and which ends with a condition for a metrizable space, but with necessary and sufficient conditions somewhere between these extremes for semi-metric spaces and Moore spaces?

THEOREM (Moore) $)^{7}$ A necessary and sufficient condition that a space $S$ satisfying Axiom 0 of [7] be metrizable is that there exist a sequence $\left\{K_{i}\right\}$ such that (1) for each natural number $n, K_{n}$ is a collection of regions in $S$ covering $S$ and (2) if $p$ is a point, $q$ is a point distinct from $p$, and $R$ is a region containing $p$, then there exists a natural number $n$ such that if each of the letters $h$ and $k$ denotes an element of $K_{n}, g \supset p$, and $g \cdot h \neq 0$, then $R-q \supset h$.

\section{REFERENCES}

1. R. H. Bing, Metrization of topological spaces, Canad. J. Math., 3 (1951), 175-186.

2. E. W. Chittenden, On the meirization problem and related problems in the theory of abstract sets, Bull. Amer. Math. Soc., 33 (1927), 13-34.

7 The terms "point" and "region" are undefined. Axiom 0 states that every region is a point set. 
3. Mary E. Estill, Concerning abstract spaces, Duke Math. J., 17 (1950), 320.

4. M. Frechet, Espaces abstracts, Paris, 1928.

5. F. B. Jones, Concerning normal and completely normal spaces, Bull. Amer. Math. Soc., 43 (1937), 671-677.

6. Ernest Michael, $A$ note on paracompact spaces, Proc. Amer. Math. Soc., 4 (1953), 831-838.

7. R. L. Moore, Foundations of point ect theory, Amer. Math. Soc. Colloquium Publications, 13, New York, 1932.

8. - - A set of axioms for plane analysis situs, Fund. Math. 25 (1935), 13-28.

9. W. Sierpinski, General topology, trans. C. Cecilia Krieger, University of Toronto Press, 1952.

10. A. H. Stone, Paracompactness and product spaces, Bull. Amer. Math. Soc., 54 (1948), 977-982.

11. C. W. Vickery, On spaces $(E)$ and Moore spaces, Bull. Amer. Math. Soc., 46 (1940), 433.

12. G: T. Whyburn, Analytic topology, Amer. Math Soc. Colloquium Publications, 28, New York, 1942.

13. $\quad$, The Boulder colloquium, Bull. Amer. Math. Soc, 35 (1929), 775-777.

14. W. A. Wilson, On semi-metric spaces, Amer. Jour. of Math., 53 (1931), 361-373.

The University of NoRTh Carolina AND

ThE UNIVERSity oF MARYLAND 


\section{PACIFIC JOURNAL OF MATHEMATICS}

EDITORS

\author{
H. L. Royden \\ Stanford University \\ Stanford, California \\ E. Hewite \\ University of Washington \\ Seattle 5 , Washington
}

\author{
R. P. Dilworth \\ California Institute of Technology \\ Pasadena 4, California
}

\author{
A. Horn* \\ University of California \\ Los Angeles 24, California
}

\section{ASSOCIATE EDITORS}
E. F. BECKENBACH
M. HALL
M. S. KNEBELMAN
J. J. STOKER
C. E. BURGESS
P. R. HALMOS
I. NIVEN
V. GANAPATHY IYER
T. G. OSTROM
G. SZEKERES
H. BUSEMANN
R. D. JAMES
M. M. SCHIFFER
F. WOLF
H. FEDERER

\section{SPONSORS}

\author{
UNIVERSITY OF BRITISH COLUMBIA \\ CALIFORNIA INSTITUTE OF TECHNOLOGY \\ UNIVERSITY OF CALIFORNIA \\ CALIFORNIA RESEARCH CORPORATION \\ MONTANA STATE UNIVERSITY \\ UNIVERSITY OF NEVADA \\ OREGON STATE COLLEGE \\ UNIVERSITY OF OREGON \\ UNIVERSITY OF SOUTHERN CALIFORNIA
}

\author{
STANFORD UNIVERSITY \\ UNIVERSITY OF UTAH \\ WASHINGTON STATE COLLEGE \\ UNIVERSITY OF WASHINGTON \\ AMERICAN MATHEMATICAL SOCIETY \\ HUGHES AIRCRAFT COMPANY
}

Mathematical papers intended for publication in the Pacific Journal of Mathematics should be typewritten (double spaced), and the author should keep a complete copy. Manuscripts may be sent to any of the editors. Manuscripts intended for the outgoing editors should be sent to their successors. All other communications to the editors should be addressed to the managing editor, Alfred Horn at the University of California, Los Angeles 24, California.

50 reprints of each article are furnished free of charge; additional copies may be obtained at cost in multiples of 50 .

The Pacific Journal of Mathematics is published quarterly, in March, June, September, and December. The price per volume (4 numbers) is $\$ 12.00$; single issues, $\$ 3.50$. Back numbers are available. Special price to individual faculty members of supporting institutions and to individual members of the American Mathematical Society: $\$ 4.00$ per volume; single issues, $\$ 1.25$.

Subscriptions, orders for back numbers, and changes of address should be sent to Pacific Journal of Mathematics, c/o University of California Press, Berkeley 4, California.

Printed at Kokusai Bunken Insatsusha (International Academic Printing Co., Ltd.), No. 10, 1-chome, Fujimi-cho, Chiyoda-ku, Tokyo, Japan.

* During the absence of E. G. Straus.

PUBLISHED BY PACIFIC JOURNAL OF MATHEMATICS, A NON-PROFIT CORPORATION COPYRIGHT 1956 BY PACIFIC JOURNAL OF MATHEMATICS 


\section{Pacific Journal of Mathematics}

\section{Vol. 6, No. $2 \quad$ December, 1956}

Louis Auslander, Remark on the use of forms in variational calculations .......................................... 209

Hubert Spence Butts, Jr. and Henry B. Mann, Corresponding residue systems in algebraic number fields ........................ 211

L. Carlitz and John Herbert Hodges, Distribution of matrices in a finite field............................................

Paul Civin and Bertram Yood, Invariant functionals ............... 231

David James Dickinson, Henry Pollak and G. H. Wannier, On a class of polynomials orthogonal over a denumerable set .................

Bernard Friedman and Luna Mishoe, Eigenfunction expansions associated with a non-self-adjoint differential equation ....................

Luna Mishoe and G. C. Ford, On the uniform convergence of a certain eigenfunction series .............................. 271

John W. Green, Mean values of harmonic functions on homothetic curves...........................................

Charles John August Halberg, Jr. and Angus E. Taylor, On the spectra of linked operators .....................................

Chuan Chih Hsiung, Some integral formulas for closed hypersurfaces in Riemannian space ................................... 291

Norman D. Lane, Differentiable points of arcs in conformal n-space 301

Louis F. McAuley, A relation between perfect separability, completeness, and normality in semi-metric spaces

G. Power and D. L. Scott-Hutton, The slow shearing motion of a liquid past a semi-infinite plane .............................

A. C. Schaeffer, Entire functions

Edward Silverman, An intrinsic inequality for Lebesgue area...

Choy-Tak Taam, Asymptotic relations between systems of differential equations.

Ti Yen, Quotient algebra of a finite $A W^{*}$-algebra ... 